\title{
REAL LINE BUNDLES ON SPHERES
}

\begin{abstract}
ALLAN L. EDELSON ${ }^{1}$
ABSTRACT. In a recent paper the author proved a classification theorem for Atiyah-real vector bundles on spaces with free involutions. This result is now applied to the group of Atiyah-real line (i.e., one-dimensional) bundles on spheres, denoted $L_{R}\left(S^{n}\right)$. It is proved that such bundles are classified by maps into a complex quadric $Q C^{n}$. Using this classification it is proved that $L_{R}\left(S^{1}\right)=0$ and that for $n \geqq 3$ the groups are all isomorphic.
\end{abstract}

1. Introduction. Atiyah-real vector bundles and the Grothendieck group $K R(X)$ were defined and studied in [1]. In [2] results were obtained concerning the classification of such bundles by equivariant maps into the complex Grassmann manifold. Specifically, it is shown that when $X$ carries a fixed point free involution there is an isomorphism $\theta:[X, B U-B O]_{\mathrm{eq} .} \rightarrow \widetilde{K R}(X)$. Here $B O$ is identified with the fixed point set of $B U$ under the involution induced by complex conjugation. The subscript denotes equivariant maps. Notice that $B U-B O$ has no fixed points.

In this paper the above classification is applied to Atiyah-real line (i.e. one-dimensional) bundles. These bundles form a group under tensor product denoted here by $L_{R}(X)$. Let $Q\left(C^{n}\right)$ denote the complex quadric with homogenous defining equation $\sum z_{i}^{2}=0$, and let $Q=U_{1 \leq n \leq \infty} Q\left(C^{n}\right)$. We then have the following:

Proposition 2. The inclusion $S^{n-1} \rightarrow S^{n}$ defines a natural bijective correspondence $\left[S^{n}, Q\right]_{\mathrm{eq}} \rightarrow\left[S^{n-1}, Q\right]_{\mathrm{eq}}$. for $n \geqq 4$.

COROLlaRY. For $n \geqq 4$ there is a natural isomorphism $L_{R}\left(S^{n}\right)$ $\rightarrow L_{R}\left(S^{n-1}\right)$.

Proposition 3. $L_{R}\left(S^{1}\right)=0$.

Throughout this paper all spaces are compact, Hausdorff. The reader is referred to [1] for the definition and properties of Atiyahreal vector bundles. The sphere $S^{n}$ is assumed to carry the antipodal

\footnotetext{
Received by the editors September 19, 1969 and, in revised form, December 22, 1969.

AMS 1970 subject classifications. Primary 55F15, 55F50.

Key words and phrases. Atiyah-real vector bundle, conjugate linear isomorphism, equivariant homotopy, line bundle.

1 This paper forms part of the author's doctoral thesis submitted in June 1968, at the State University of New York, Stony Brook.
} 
involution and the inclusion $S^{n-1} \rightarrow S^{n}$ is onto an equator. Complex conjugation will be denoted $\kappa: C^{n} \rightarrow C^{n}$.

2. Classification of real line bundles. Let $\xi \rightarrow X$ be a complex vector bundle, and $\bar{\xi}$ the complex conjugate bundle. Then the natural map $\xi \rightarrow \bar{\xi}$ is a conjugate linear isomorphism and an involution $\tau: \xi \rightarrow \xi$ defines a conjugate linear isomorphism $\xi_{x} \rightarrow \xi_{\tau(x)}$. An involution is defined on $\bar{\xi}$ by requiring that the following diagram commute:

$$
\begin{aligned}
\xi_{x} & \rightarrow \bar{\xi}_{x} \\
\downarrow & \tau \quad \downarrow \\
\xi_{\tau(x)} & \rightarrow \bar{\xi}_{\tau(x)}
\end{aligned}
$$

The tensor product of two real bundles is again a real line bundle. We require the following extension of a well-known result about complex vector bundles:

Lemma 1. Let $\xi \rightarrow X$ be a real line bundle. Then $\xi \otimes \bar{\xi}$ is the trivial real line bundle over $X$.

Proof. The standard metric on the classifying bundle is invariant and hence the metric induced in $\xi$ is equivariant in the sense that if $\mu: \xi \otimes \xi \rightarrow C$ is this metric, then $\mu(\bar{u}, \bar{v})=\kappa(\mu(u, v))$.

Define a vector bundle morphism $f: \xi \otimes \xi \rightarrow X \times C$ by $f\left(u_{x} \otimes v_{x}\right)$ $=\left(x, \mu\left(u_{x}, v_{x}\right)\right)$. Then $f$ is a surjective morphism of one-dimensional vector bundles and hence is an isomorphism of the underlying complex vector bundles. Since $f$ is equivariant it is an isomorphism of real vector bundles.

Corollary. The real line bundles over $X$ form a group under tensor products.

Let $L_{R}(X)$ denote this group. By Proposition II.I of [2] there is a natural bijective correspondence $L_{R}(X) \leftrightarrow\left[X, P C^{\infty}\right]_{\text {eq. }}$, and if $X$ carries a fixed-point free involution there is, by Proposition II.2 of [2], a natural bijective correspondence $L_{R}(X) \rightarrow\left[X, P C^{\infty}-P R^{\infty}\right]_{\text {eq. }}$. Let $Q\left(C^{n}\right)$ denote the quadric surface in $P C^{n}$ defined by $\sum_{1}^{n} z_{i}^{2}=0$. Then $Q\left(C^{n}\right)$ is invariant under the involution and has no fixed points. It is proved in [3] that $P R^{n}$ is a deformation retract of $P C^{n}-Q C^{n}$ in the category of real spaces. Since $P C^{n} / P R^{n}$ is homeomorphic to the Thom space of the normal bundle to $Q\left(C^{n}\right)$ in $P C^{n}$ as real spaces with basepoint (see [3, Corollary 6.4$]$ ), by retracting along the fibres we have the following:

Lemma 2. $Q\left(C^{n}\right)$ is a deformation retract of $P C^{n}-P R^{n}$ as spaces with involution. 
The inclusions $\left(P C^{n}-P R^{n}\right) \rightarrow\left(P C^{n+1}-P R^{n+1}\right)$ induce inclusions $Q\left(C^{n}\right) \rightarrow Q\left(C^{n+1}\right)$ and commute with the deformation retractions. Hence if we define $Q=U_{1 \leq n \leq \infty} Q\left(C^{n}\right), Q$ is a deformation retract of $P C^{\infty}-P R^{\infty}$ as spaces with involution.

COROLlaRY. There is a natural bijective correspondence

$$
\left[X, P C^{\infty}-P R^{\infty}\right]_{\text {eq. }}=[X, Q]_{\text {oq. }} .
$$

\section{Real line bundles on spheres.}

Proposition 1. The inclusion i: $P C^{\infty}-P R^{\infty} \rightarrow P C^{\infty}$ is a weak homotopy equivalence.

Proof. Consider the fibration $S^{1} \rightarrow S^{2 n-1} \stackrel{p}{\rightarrow} P C^{n}$ where $S^{2 n-1}$ is viewed as the unit sphere in $C^{n}$ and $p$ is the projection. Since $p$ is equivariant, $p^{-1}\left(P R^{n}\right)=S^{2 n-1} \cap R^{n}$, where $R^{n}$ is viewed as the fixed point set of $C^{n}$. We then have the restriction

$$
S^{1} \rightarrow S^{2 n-1}-S^{n-1} \stackrel{p}{\rightarrow} P C^{n}-P R^{n} .
$$

If we view $S^{2 n-1}$ as the unit sphere in $R^{2 n}$ then a point of $S^{2 n-1}$ is a pair $(X, Y), X, Y \in R^{n}$, and $|X|^{2}+|Y|^{2}=1$. We can view $S^{n-1}$ as the subset $\left\{(X, Y) \in S^{2 n-1} \mid Y=0\right\}$. Then $\left\{(X, Y) \in S^{2 n-1} \mid X=0\right\}$; also an $n-1$ sphere is a strong deformation retract of $S^{2 n-1}-S^{n-1}$. Explicitly, the homotopy may be defined by

$$
h_{t}(X, Y)=(t \cdot X, f(t, Y) \cdot Y)
$$

where

$$
(f(t, Y))^{2}=\left(1-t^{2}+t^{2}|Y|^{2}\right) /|Y|^{2} .
$$

It then follows from the exact homotopy sequences of the above fibrations that the inclusion $i: P C^{n}-P R^{n} \rightarrow P C^{n}$ defines an isomorphism $i_{*}: \pi_{k}\left(P C^{n}-P R^{n}\right) \rightarrow \pi_{k}\left(P C^{n}\right)$ for $k \leqq n-2$.

Since $P C^{n+1}$ is obtained from $P C^{n}$ by adjoining a single cell of dimension $2 n$, the inclusion $P C^{n} \rightarrow P C^{n+m}$ induces an isomorphism $\pi_{k}\left(P C^{n}\right) \rightarrow \pi_{k}\left(P C^{n+m}\right)$ for $k \leqq 2 n-1$. From the commutative diagram

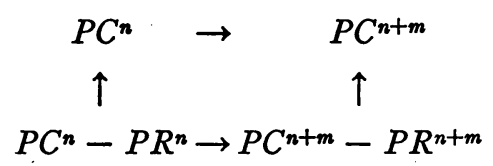

it follows that the inclusion $P C^{n}-P R^{n} \rightarrow P C^{n+m}-P R^{n+m}$ induces an isomorphism $\pi_{k}\left(P C^{n}-P R^{n}\right) \rightarrow \pi_{k}\left(P C^{n+m}-P R^{n+m}\right)$ for $k \leqq n-2$. Thus by Proposition 4.3 of [4] the inclusions $P C^{n} \rightarrow P C^{\infty}$ and $P C^{n}-P R^{n}$ $\rightarrow P C^{\infty}-P R^{\infty}$ induce isomorphisms in homotopy for $k \leqq n-2$. Letting 
$n$ go to infinity it follows that $i_{*}: \pi_{k}\left(P C^{\infty}-P R^{\infty}\right) \rightarrow \pi_{k}\left(P C^{\infty}\right)$ is an isomorphism for all $k$. This proves Proposition 1.

Proposition 2. Let $\Psi:\left[S^{n}, Q\right]_{\text {eq. }} \rightarrow\left[S^{n-1}, Q\right]_{\text {eq. }}$ be the map induced by the inclusion $S^{n-1} \rightarrow S^{n}$. Then $\Psi$ is injective for $n \geqq 3$ and surjective for $n \geqq 4$.

Proof. If $f: S^{n} \rightarrow Q$ is equivariant then so is its restriction to $S^{n-1}$. Any equivariant homotopy $h: S^{n} \times I \rightarrow Q$ has an eq. (= equivariant) restriction to $S^{n-1} \times I$. Let $D_{n}^{+}$and $D_{n}^{-}$denote the upper and lower hemispheres of $S^{n}$, and $\tau$ the antipodal map. The $n$-skeleton $\left(D_{n}^{-} \times I\right)_{n}$ $=D_{n}^{-} \times\{0,1\} \cup S^{n-1} \times I$. There is a map $\alpha:\left(D_{n}^{-} \times I\right)_{n} \rightarrow Q$ defined by

$$
\begin{array}{ll}
\alpha(x, 0)=f(x), & x \in D_{\bar{n}}^{-}, \\
\alpha(x, 1)=g(x), & x \in D_{\bar{n}}^{-}, \\
\alpha(x, \tau)=h(x, \tau), & x \in S^{n-1} .
\end{array}
$$

Clearly these definitions agree on the intersections. Then for $n \geqq 3$, $\alpha$ extends, by Proposition 1, to

$$
\begin{aligned}
& \alpha^{\prime}(x, \tau)=h(x, \tau), \quad x \in S^{n-1}, \\
& \alpha^{\prime}(x, 0)=f(x), \quad x \in D_{n}^{-}, \\
& \alpha^{\prime}(x, 1)=g(x), \quad x \in D_{\bar{n}}^{-} .
\end{aligned}
$$

We now wish to extend $\alpha^{\prime}$ to an equivariant homotopy $S^{n} \times I \rightarrow Q$ whose restrictions to $S^{n} \times\{0\}$ and $S^{n} \times\{1\}$ are the given maps $f$ and $g$. We do this using the involution in $Q$. Define a map $\alpha^{\prime \prime}: S^{n} \times I \rightarrow Q$ by the following:

$$
\begin{array}{llrl}
\alpha^{\prime \prime}(x, \tau) & =\alpha^{\prime}(x, \tau), & & x \in D_{n}^{-}, \\
\alpha^{\prime \prime}(x, \tau) & =\kappa\left(\alpha^{\prime}(\tau(x), \tau)\right), & & x \in D_{n}^{+} .
\end{array}
$$

If $x \in S^{n-1}$, then $\alpha^{\prime \prime}(x, \tau)=\alpha^{\prime}(x, \tau)=h(x, \tau)$. Furthermore $\alpha^{\prime \prime}(x, 0)$ $=\alpha^{\prime}(x, 0)=f(x)$ for $x \in D_{n}^{-}$and for $x \in D_{n}^{+}$we have $\alpha^{\prime \prime}(x, 0)$ $=\kappa\left(\alpha^{\prime}(\tau(x), 0)\right)=\kappa(f(\tau(x)))=f(x)$ since $f$ is equivariant. Thus $\alpha^{\prime \prime}$ restricted to $S^{n} \times\{0\}$ agrees with $f$ and by a similar agreement $\alpha^{\prime \prime}$ restricted to $S^{n} \times\{1\}$ agrees with $g$. This shows that $\psi$ is one-to-one.

Now let $f: S^{n-1} \rightarrow Q$ be equivariant. By Proposition $1, f$ extends to a map $f^{\prime}: D_{n}^{-} \rightarrow Q$, at least for $n \geqq 4$. Define a map $f^{\prime \prime}: S^{n} \rightarrow Q$ by the following:

$$
\begin{aligned}
& f^{\prime \prime}(x)=f^{\prime}(x), \quad x \in D_{\bar{n}}^{-}, \\
& f^{\prime \prime}(x)=\kappa\left(f^{\prime}(\tau(x))\right), \quad x \in D_{n}^{+} .
\end{aligned}
$$

Then if $x \in D_{n}^{-}$, 


$$
f^{\prime \prime}(\tau(x))=\kappa\left(f^{\prime}(x)\right)=\kappa\left(f^{\prime \prime}(x)\right)
$$

and if $x \in D_{n}^{+}$,

$$
f^{\prime \prime}(\tau(x))=f^{\prime}(\tau(x))=\kappa\left(f^{\prime \prime}(x)\right)
$$

so that $f^{\prime \prime}$ is equivariant and agrees with $f$ on $S^{n-1}$. This implies $\psi\left[f^{\prime \prime}\right]=[f]$ and that for $n \geqq 4, \psi$ is onto. This proves Proposition 2 . By the naturality of tensor products $\psi$ is in fact a homomorphism, hence we have:

Corollary. For $n \geqq 4$ the inclusion map defines an isomorphism

$$
L_{R}\left(S^{n}\right) \cong L_{R}\left(S^{n-1}\right) \text {. }
$$

Proposition 3. $\left[S^{1}, Q\right]_{\text {eq. }}=0$.

Proof. Let $f, g: S^{1} \rightarrow Q$ be equivariant. Let $S^{0}=\left\{x_{+}, x_{-}\right\} \subset S^{1}$. There is a path $\sigma: I \rightarrow Q$ with $\sigma(0)=f\left(x_{+}\right), \sigma(1)=g\left(x_{+}\right)$. Then the restrictions of $f$ and $g$ to $D_{1}^{+}$, together with $\sigma(I)$ and $\kappa(\sigma(I))$, define a map $h: S^{1} \rightarrow Q$ which extends to $D_{2}^{+}$. The extension $h\left(D_{2}^{+}\right)$and its conjugate, $\kappa\left(h\left(D_{2}^{+}\right)\right)$, define an equivariant homotopy $f \simeq g$ as in the proof of Proposition 2. This completes the proof of Proposition 3.

NotE. The referee has pointed out that it follows from results of J. Levine, Spaces with involutions and bundles over $P^{n}$, Amer. J. Math. 85 (1963), that $L_{R}\left(S^{2}\right)$ is infinite cyclic and $L_{R}\left(S^{n}\right)=0$ for $n>2$.

Levine's equivariant homotopy group $\pi_{n}^{0}(X ; T)=\left[S^{n}, A ; X, T\right]_{\text {eq }}$. is precisely $L_{R}\left(S^{n}\right)$ when $X=Q$. With the use of his exact sequence (Theorem 4.3) and the formula on p. 527, the groups can be computed.

\section{BIBLIOGRAPHY}

1. M. F. Atiyah, K-theory and reality, Quart. J. Math. Oxford Ser. (2) 17 (1966), 367-386. MR 34 \#6756.

2. A. L. Edelson, Real vector bundles and spaces with free involutions, Trans. Amer. Math. Soc. (to appear).

3. P. Landweber, Fixed point free conjugations on complex manifolds, Ann. of Math. (2) 86 (1967), 491-502. MR 36 \#3382.

4. D. Husemoller, Fibre bundles, McGraw-Hill, New York, 1966. MR 37 \#4821.

State University of New York, Stony Brook, New York 11790

University of California, Davis, California 95616 